${ }^{1}$ Physikalisch-Technische Bundesanstalt (Bundesallee 100, 38116 Braunchweig, Germany)

${ }^{2}$ Kyiv National Taras Shevchenko University, Faculty of Physics

(64/13, Volodymyrs'ka Str., Kyiv 01601,Ukraine; e-mail: mykhailo.rakov@univ.kiev.ua)

\title{
NUMERICAL STUDIES OF ENTANGLEMENT PROPERTIES IN ONE- AND TWO-DIMENSIONAL PACS 64.70.Tg, 03.67.Mn QUANTUM ISING AND XXZ MODELS
}

\begin{abstract}
We investigate entanglement properties of infinite one- and two-dimensional spin-1/2 quantum Ising and XXZ models. Tensor network methods (TI MPS with single-site update and TEBD for MPS, TERG and CTMRG with "simple update" for PEPS) are used to model the ground states of the studied models. Different entanglement measures, such as the one-site entanglement entropy, one-tangle, concurrence of formation and assistance, negativity and entanglement per bond are compared with respect to their ability to exhibit "structures" in the phase diagram of the models (e.g., phase transitions). We study the connection between symmetries and the entanglement of ground states and analyze short- and long-range entanglement through the entanglement monogamy.
\end{abstract}

Ke ywords: quantum phase transitions, quantum entanglement, tensor networks.

\section{Introduction}

Matter comes in different phases, and usually one can switch between them by changing the temperature. Close to zero temperature, thermal fluctuations disappear, and quantum fluctuations dominate. In this case, by changing an appropriate control parameter, one can induce quantum phase transitions (QPTs) between the different phases of quantum systems. QPTs occur in many different physical systems, and they attract a lot of attention in condensedmatter physics [1].

The reason for the recent surge of interest in QPTs are new and exotic quantum phases and critical points, which cannot be described within Landau's theory of phase transitions, i.e. they cannot be characterized by an order parameter. Examples are topologically ordered phases [2], quantum spin liquids [3], or deconfined quantum critical points [4].

At and close to critical points, different parts of the system are quantum mechanically strongly correlated, and various correlation functions may show singular behavior [5]. For decades, quantum manybody systems were studied in terms of such correla-

(C) B. BRAIORR-ORRS, M. WEYRAUCH,

M.V. RAKOV, 2016

ISSN 2071-0194. Ukr. J. Phys. 2016. Vol. 61, No. 7 tion functions within Landau's theory. Several years ago, in quantum information theory, it was suggested that quantum phases and QPTs, as well as other properties of many-body systems, can be characterized and distinguished in terms of the quantum entanglement [6, 7]. Simple entanglement quantifiers may reveal the essential structure of many-body systems, and the present work starts from this point of view.

We study entanglement properties of the ground states of one- (1D) and two-dimensional (2D) spin$1 / 2$ models using different entanglement quantifiers. The ground states are generated, by using tensor-network techniques. Moreover, a study of the Coffman-Kundu-Wootters (CKW) inequality [8] enables some judgment on the entanglement distribution within the many-body wave function [9].

Numerous different entanglement quantifiers have been proposed [10]. Those which are studied here [5, 11-14] are listed in the Appendix. Studies of the entanglement properties of the many-body systems mainly use the entanglement entropy, the one-tangle, the concurrence, and the fidelity [5]. There are also investigations of the multipartite entanglement properties of the states (e.g., tripartite entanglement [15] and global entanglement [14]). 
In the present paper, we focus on ground states of the quantum Ising model and of the anisotropic XXZ model in $1 \mathrm{D}$ and $2 \mathrm{D}$. We will analyze how a broken symmetry reveals itself in the entanglement quantifiers. Investigations in this direction were done in $[16,17]$ based on the concurrence of formation, and we extend this analysis with numerical tools to other measures. We show analytically and numerically that - in contrast to the concurrence of formation - the concurrence of assistance shows U(1)symmetry breaking and confirm this with numerical results. An analysis of the entanglement monogamy enables us to analyze the entanglement distribution on the basis of the CKW inequality. We obtain that, close to some critical points, the most entanglement is not contained in the nearest neighbour entanglement. We compare our results with those based on other techniques [11-13] whenever possible.

In order to simulate the ground states of $1 \mathrm{D}$ and $2 \mathrm{D}$ spin models we use a tensor network (TN) approach $[18,19]$. TN methods became very popular in recent years for the simulation of strongly correlated systems. They proved to be rather flexible in various aspects: one can study a variety of systems in different dimensions; of finite or infinite size; with different boundary conditions, symmetries; systems of bosons, fermions and (frustrated) spins. For a recent review, see Ref. [20]. The basic idea of TN methods is to represent the wave function of a many-body quantum system by a network of interconnected tensors. Matrix product states (MPS) are the most famous among the TN states [19]. Powerful algorithms such as the Density Matrix Renormalization Group (DMRG) [21] or Time-Evolving Block Decimation (TEBD) [22] can be formulated in terms of MPS. The two-dimensional generalization of the matrix product states is called projected entangled pair states (PEPS) [23]. Details about the PEPS and the MPS can be found in Refs. [24-27].

We use techniques, which are able to treat models in the thermodynamic limit: MPS [27] in $1 \mathrm{D}$ and the Tensor-Entanglement Renormalization Group (TERG) [28], the Corner Transfer Matrix Renormalization Group (CTMRG) [29, 30] for PEPS in $2 \mathrm{D}$. More specifically, we use the imaginary-time evolution (the TI MPS with one-site update [31,32], the TEBD for MPS [33] in 1D, and the "simple update" scheme [25] for PEPS in 2D) to find the approximate ground states for the models under investi- gation. Exploiting the translational invariance of the ground states enables algorithms with reasonable requirements for computational resources [28, 34].

This paper is organized as follows. In Section 2, we briefly describe numerical algorithms we use and give appropriate references. Section 3 presents numerical results and their interpretations for the quantum Ising and XXZ models. Conclusions are made in Section 4. Various entanglement measures are briefly listed and discussed in the Appendix.

\section{Ground State Calculation}

In our studies, we use four different tensor network algorithms: TI MPS and TEBD for MPS in one dimension and TERG and CTMRG with "simple update" in two dimenions. These methods are well established, and we do not present details here, but refer the reader to the literature cited in Introduction. Two-dimensional models are investigated on a square lattice. Both $1 \mathrm{D}$ and $2 \mathrm{D}$ simulations are performed for periodic boundary conditions.

The MPS representation of a wave function $|\Psi\rangle$ for $N$ spins

$|\Psi\rangle=\operatorname{tTr}\left\{A_{k_{i} l_{i}}^{\sigma_{i}} A_{k_{j} l_{j}}^{\sigma_{j}} \ldots\left|\sigma_{i} \sigma_{j} \ldots\right\rangle\right\}$.

is written in terms of rank-3 tensors $\left(A_{k l}^{\sigma}\right)$ with physical (spin) index $\sigma$ of size two (since we consider spin$1 / 2$ systems only) and virtual dimensions $k, l$ of size $m$. The tensor trace tTr includes the summation over all spin configurations and over all bond indices.

All our methods utilize the imaginary-time evolution based on a Trotter expansion [35] of the evolution operator for finding the ground-state wave functions [36]. Probably, the best known algorithm for the 1D imaginary-time evolution is the TEBD algorithm proposed by Vidal [33]. The TEBD algorithm naturally leads to a ground state in the canonical form of MPS [22,37]. In this form, besides the tensors $A$ at each site, one also has bond vectors $\boldsymbol{\lambda}$ at each bond of the tensor network. Within the given canonical MPS, the bond states (bond vectors) can be regarded as renormalized bases of the physical degrees of freedom of the many-body system (e.g. "effective" spins). It is known that TEBD breaks the translational symmetry and leads to a bipartition of the tensor network into two sublattices. The TI MPS method [38] maintains the translational invariance exactly, i.e. quantum states are represented by an MPS

ISSN 2071-0194. Ukr. J. Phys. 2016. Vol. 61, No. 7 
with identical matrices $A^{\sigma}$ at each lattice site. In their simplest form, both TEBD and TI MPS algorithms do not preserve symmetries of the Hamiltonian.

Projected entangled pair states (PEPS) are 2D generalizations of the MPS. A random wave function $|\psi\rangle$ PEPS representation is constructed from a product of equal rank-5 tensors $A$ at the lattice sites $i, j, \ldots$,

$|\Psi\rangle=\operatorname{tTr}\left\{A_{k_{i} l_{i} m_{i} n_{i}}^{\sigma_{i}} A_{k_{j} l_{j} m_{j} n_{j}}^{\sigma_{j}} \ldots\left|\sigma_{i} \sigma_{j} \ldots\right\rangle\right\}$.

The size of each physical (spin) index $\sigma$ is two, since we consider spin- $1 / 2$ systems only. The size of each virtual bond $k, l, m, n$ is $D$. The tensor trace $\mathrm{tTr}$ includes the summation over all spin configurations and over all bond indices.

Generalization of the TEBD algorithm to two dimensions leads to the so-called "simple update" scheme $[25,39]$ for the imaginary-time evolution. Unfortunately, no exact canonical form for PEPS exists, but the "simple update" algorithm for the imaginarytime evolution leads to a ground state in the approximate canonical form of PEPS.

TERG is based on the tensor renormalization group (TRG) method introduced by Levin and Nave [34] for classical systems. It was modified for quantum systems in Ref. [28] with the use of the concept of "impurity" tensors. In its original formulation, TERG enables to calculate two-site and one-site observables for a given PEPS. In our implementation, we modified the TERG algorithm in order to determine four-site reduced density matrices for a given PEPS. The accuracy of the TERG is controlled by a cutting parameter $D_{c}$, which defines the size of the tensors during the renormalization procedure.

The corner transfer matrix renormalization group (CTMRG) was first introduced by Baxter [40]. It was further developed and applied to classical statistical systems by Nishino and Okunishi [41, 42]. More recently, it was adapted to the contraction of tensor networks by Orus [30, 43]. From a given PEPS, the CTMRG determines the "environment tensor" of a block of the four sites. The locations of these four sites correspond to the locations of the "impurity sites" in TERG. With the "environment tensor," it is easy to obtain the four-spin reduced density matrix.

Almost all entanglement characteristics we calculate are obtained from one-site and two-site reduced density matrices. Only the bipartite entanglement per bond is obtained directly from the tensor network representation of the state. The definitions and corresponding formulas for the entanglement quantifiers are briefly presented in the Appendix.

Numerical results obtained with the TEBD algorithm typically agree with TI MPS results, and TERG results agree with CTMRG calculations, if the parameters of calculations are properly controlled.

\section{Entanglement Quantifiers and Entanglement Distribution: Numerical Results and Physical Interpretation}

In this section, we use the methods mentioned in the previous section to analyze $1 \mathrm{D}$ and $2 \mathrm{D}$ spin- $1 / 2$ systems: the quantum Ising model in a transverse magnetic field and the XXZ model. We calculate various entanglement measures for these systems such as the one-site entanglement entropy, one-tangle, concurrence of formation and negativity. Furthermore, we determine bounds on the localizable entanglement in terms of the concurrence of assistance and maximal two-point correlation functions, local entanglement, and entanglement per bond. We compare these quantities and discuss their ability to identify critical points and distinguish between different phases. The mentioned entanglement measures are briefly defined in the Appendix.

An interesting characteristic we analyze using the calculated entanglement measures is the monogamy of entanglement [8] or - more precisely - the entanglement distribution between different parties. Somewhat naively, the entanglement monogamy may be expressed as follows: if two parties are maximally entangled, they cannot be entangled at all with a third party. Expressions for the distribution of entanglement in the form of monogamy relations for multiqubit systems, based on the concurrence of formation $C_{F}$ and the concurrence of assistance $C_{A}$ have been obtained in Refs. [44, 45]. Thus, among the different entanglement measures we calculate in the present work, the concurrences of formation and assistance are of primary interest. For the models studied in the present paper, a naive entanglement monogamy analysis was done in Ref. [11], by using Monte-Carlo methods for the calculation of $C_{F}$. Here, we provide a more comprehensive analysis based on monogamy relations for $C_{F}$ and $C_{A}$.

Entanglement monogamy relations for $N$-qubit systems are obtained from the Coffman-Kundu- 
Wootters (CKW) [8] inequality

$\left[C_{F}\right]_{A \mid B_{1} B_{2} \ldots B_{N-1}}^{2} \geq\left[C_{F}\right]_{A B_{1}}^{2}+$

$+\left[C_{F}\right]_{A B_{2}}^{2}+\ldots+\left[C_{F}\right]_{A B_{N-1}}^{2}$,

where $\left[C_{F}\right]_{A B_{i}}=\left[C_{F}\right]\left(\rho_{A B_{i}}\right)$ is the concurrence of the reduced density matrix $\rho_{A B_{i}}$ and $\left[C_{F}\right]_{A \mid B_{1} B_{2} \ldots B_{N-1}}=$ $=C\left(|\psi\rangle_{A \mid B_{1} B_{2} \ldots B_{N-1}}\right)$ the concurrence of the pure state $|\psi\rangle$ as defined in Ref. [46]. For pure $N$-qubit states, $C_{A \mid B_{1} B_{2} \ldots B_{N-1}}^{2}$ can be obtained from the onesite reduced density matrix, and it is equal to the one-tangle $\tau_{1}:\left[C_{F}\right]_{A \mid B_{1} B_{2} \ldots B_{N-1}}^{2}=4 \operatorname{det} \rho_{A}=\tau_{1}[46]$.

In our analysis, we use two main assumptions concerning the entanglement structure of the ground states of the models we study. The first is that only the nearest neighbor concurrences give major contributions to the sum of the right-hand side of (3). The larger the separation between two particles, the smaller is the concurrence between them. The second assumption is a consequence of the translational symmetry of the ground states. As a consequence, all nearest neighbor concurrences are equal.

Taking these two features of the systems under consideration into account allows us to rewrite inequality (3) for $1 \mathrm{D}$ and $2 \mathrm{D}$ models. For $1 \mathrm{D}$ systems, one obtains

$\tau_{1}^{1 \mathrm{D}} \geq 2\left[C_{F}^{1 \mathrm{D}}\right]_{\mathrm{NN}}^{2}+\delta_{F}^{1 \mathrm{D}}$,

where $\left[C_{F}^{1 \mathrm{D}}\right]_{\mathrm{NN}}^{2}$ is the nearest neighbor $(\mathrm{NN})$ concurrence of formation, and the quantity $\delta_{F}^{1 \mathrm{D}}$ contains all other bipartite concurrences. Analogously in 2D, one finds

$\tau_{1}^{2 \mathrm{D}} \geq 4\left[C_{F}^{2 \mathrm{D}}\right]_{\mathrm{NN}}^{2}+\delta_{F}^{2 \mathrm{D}}$.

Using this inequality, we check the consistency of our numerical data. Of course, the total sum of all two-particle entanglement approaches $\tau_{1}$ only in special circumstances. It is known that the many-qubit CKW inequality can be sharpened by adding threeway entanglement terms (as, e.g., in Ref. [47]). In general, the sharpening of the inequality would require taking many other many-way entanglement terms into account.

Using the concept of "sharpened" CKW inequality, we analyze the entanglement distribution in the state. In our analysis, we treat the NN two-particle entanglement terms as a short-range entanglement and all various possible other terms (contained in $\left.\delta_{F}\right)$ as the sum of many-way and long-range entanglements. Thus, the difference between $\tau_{1}$ and NN entanglements can be related to either many-way and/or long-range entanglement in the entanglement distribution. The value $\tau_{1}$ we call total entanglement to underline its role in the entanglement distribution analysis.

Note that an entanglement measure called twotangle $\tau_{2}$ exists [48]. Two-tangle is related to the concurrence of formation: $\tau_{2}=C_{F}^{2}$. The entanglement distribution analysis based on the comparison of $\tau_{1}$ and $\tau_{2}$ was already done for various onedimensional models $[9,49,50]$ and is less presented for two-dimensional quantum models $[51,52]$. Our work aims to contribute in this direction, in particular, in the monogamy analysis of two-dimensional quantum models, but we analyze one-dimensional models either.

The inequality dual to the CKW one yields the following relation which involves the concurrence of assistance $C_{A}$ on the right-hand side $[44,45]$

$$
\begin{gathered}
{\left[C_{F}\right]_{A \mid B_{1} B_{2} \ldots B_{N-1}}^{2} \leq\left[C_{A}\right]_{A B_{1}}^{2}+} \\
+\left[C_{A}\right]_{A B_{2}}^{2}+\ldots+\left[C_{A}\right]_{A B_{N-1}}^{2}
\end{gathered}
$$

Again, we introduce the quantity $\delta_{A}^{1 \mathrm{D}}$,

$\tau_{1}^{1 \mathrm{D}} \leq 2\left[C_{A}^{1 \mathrm{D}}\right]_{\mathrm{NN}}^{2}+\delta_{A}^{1 \mathrm{D}}$,

where $\left[C_{A}^{1 \mathrm{D}}\right]_{\mathrm{NN}}^{2}$ contains the nearest neighbor terms and $\delta_{A}$ the longer-ranged bipartite concurrences. In $2 \mathrm{D}$, we have

$\tau_{1}^{2 \mathrm{D}} \leq 4\left[C_{A}^{2 \mathrm{D}}\right]_{\mathrm{NN}}^{2}+\delta_{A}^{2 \mathrm{D}}$.

The comparison of $\tau_{1}$ and the entanglement covered by $C_{A}$ is another way of the gaining of information about the entanglement structure of the state.

\subsection{Quantum Ising model in a transverse field}

The spin- $\frac{1}{2}$ Ising model in a transverse magnetic field $h$ is given by the Hamiltonian

$H^{\mathrm{Ising}}=J \sum_{\langle i, j\rangle} \sigma_{i}^{z} \otimes \sigma_{j}^{z}+h \sum_{i} \sigma_{i}^{x}$

ISSN 2071-0194. Ukr. J. Phys. 2016. Vol. 61, No. 7 
where $\sigma_{i}^{\alpha}(\alpha=x, y, z)$ are the standard Pauli spin operators. This model is $Z_{2}$ symmetric (spin-flip symmetric).

The sign of the coupling constant $J$ determines the type of the interaction between spins: antiferromagnetic for $J>0$ and ferromagnetic for $J<0$. The calculated physical quantities are symmetric with respect to $J=0$. Quantities like the magnetization $m_{x}$ $(J<0)$ are mapped to their staggered counterparts $(J>0)$. In the present paper, we choose the energy scale by setting $J=-1$.

In $1 \mathrm{D}$, this model can be solved analytically using a Jordan-Wigner transformation [53]. It is well known that, at the critical points $h= \pm 1$, this model shows quantum phase transitions separating a magnetically ordered phase $(-1<h<1)$ from paramagnetic phases $(h<-1$ and $h>1)$. In the ordered phases, the $Z_{2}$ symmetry is spontaneously broken. At the critical points and in the thermodynamic limit, the ground state energy per site is given by $E_{0}=-4 / \pi$.

The 2D quantum Ising model cannot be solved analytically, and various methods are applied to solve it numerically, notably rather resource-intensive MonteCarlo (MC) methods. Such calculations find a transition between a ferromagnetic and a paramagnetic phase at a critical point $h_{\mathrm{cr}}=3.044$ [54]. The tensor network implementation we use here produces numerical results significantly faster than MC calculations, however, with less precision: our implementation determines a critical point at $h_{\mathrm{cr}} \approx 3.28$, which is determined from a singular point of the second derivative of the ground-state energy as a function of $h$. Of course, significantly more precise results could be obtained with more elaborate tensor network implementations and larger bond sizes [20]. However, it is our goal to investigate correlations and entanglement properties, by using small numerical cost. In practice, we study positive $h$ only and obtain results for negative $h$ by a reflection at $h=0$. In order to compare numerical results for one- and two-dimensional systems, we rescale the magnetic field dependence $h / h_{\mathrm{cr}}$ such that phase transitions always occur at $h / h_{\mathrm{cr}}=1$.

For $h \ll h_{\mathrm{cr}}$, the system (both in 1D and 2D) is a classical Ising model with a doubly degenerate ground state (in the thermodynamic limit). In experimental situations, this degeneracy is broken, and this is done intrinsically in our MPS and PEPS implementations as well. For $h \gg h_{\mathrm{cr}}$, the magnetic field dominates,

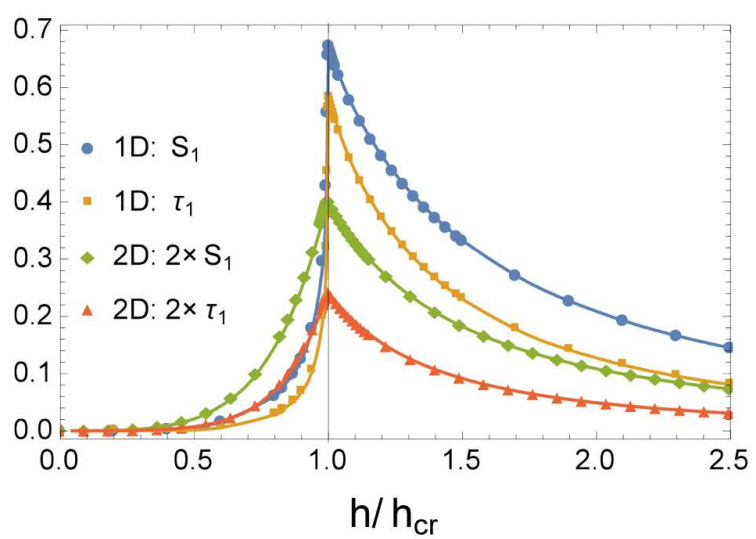

Fig. 1. One-site entanglement entropy $S_{1}$ and one-tangle $\tau_{1}$ as a function of the magnetic field $h / h_{\mathrm{cr}}$ for the $1 \mathrm{D}$ and $2 \mathrm{D}$ quantum Ising models. 2D results are multiplied by a factor of 2 . Parameters for the 1D MPS calculation: $m=20$. Parameters for the 2D TERG calculation: $D=4, D_{c}=20$

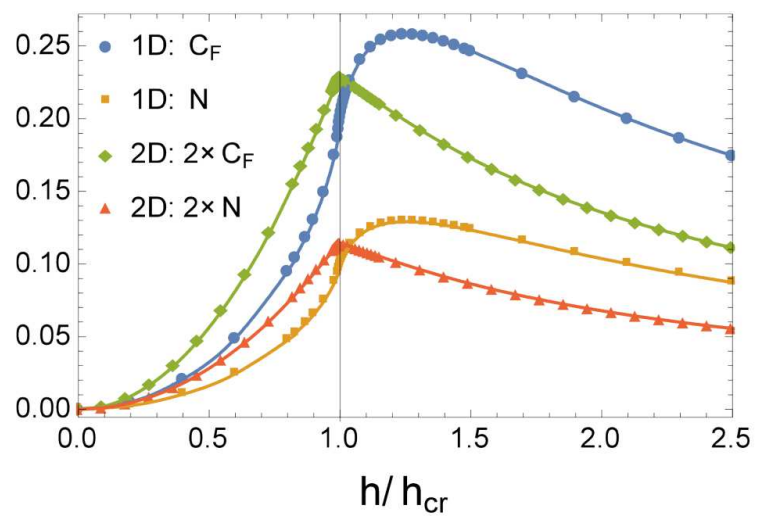

Fig. 2. Concurrence of formation $C_{F}$ and negativity $N$ as a function of the magnetic field $h / h_{\mathrm{cr}}$ for $1 \mathrm{D}$ and $2 \mathrm{D}$ quantum Ising models. 2D results are multiplied by a factor of 2 . Parameters for the $1 \mathrm{D}$ MPS calculation: $m=20$. Parameters for the 2D TERG calculation: $D=4, D_{c}=20$

and the ground state corresponds to free spins oriented according to the magnetic field.

In Fig. 1, we show the entanglement measures calculated from the single-spin density matrix: one-site entanglement entropy $S_{1}$ and one-tangle $\tau_{1}$ for the one- and two-dimensional Ising models. It is clearly seen that all these measures nicely peak in cusps at the critical point. All $2 \mathrm{D}$ results are multiplied by a factor of 2 for easier comparison.

In Figs. 2 and 3, we show entanglement measures calculated from the two-spin density matrix as a function of the magnetic field: the concurrence of forma- 


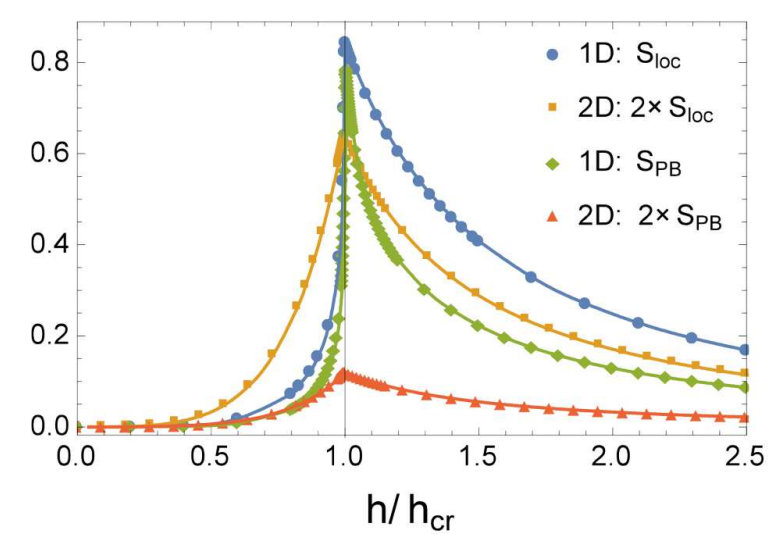

Fig. 3. Comparison of local entanglement $S_{\text {loc }}$ dependence on the magnetic field $h$ for the $1 \mathrm{D}$ and 2D quantum Ising models. Entanglement per bond $S_{\mathrm{PB}}$ dependence for the 2D Ising model. Results are renormalized to the $h / h_{\mathrm{cr}}$ dependence. Results for the 2D model are multiplied by a factor of 2 . Parameters for the 1D MPS calculation: $m=20$. Parameters for the 2D TERG calculation: $D=4, D_{c}=20$

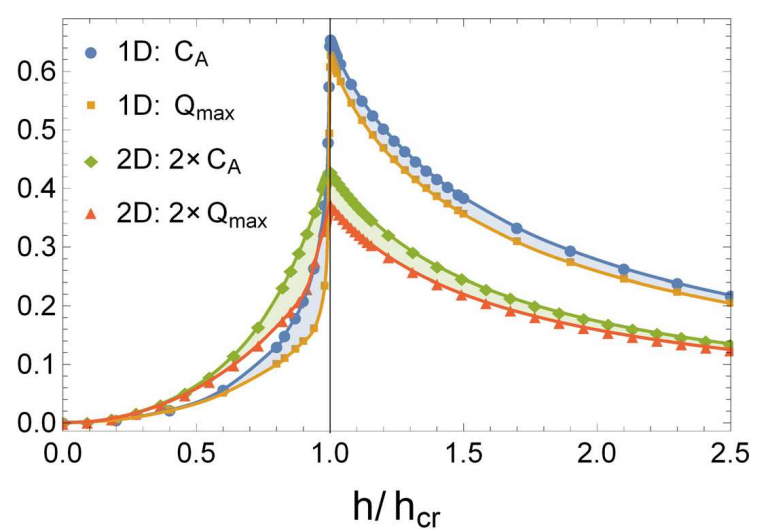

Fig. 4. Upper and lower bounds of the localizable entanglement as a function of the magnetic field $h / h_{\mathrm{cr}}$ for the $1 \mathrm{D}$ and $2 \mathrm{D}$ quantum Ising models; $2 \mathrm{D}$ results are multiplied by a factor of 2. The shaded areas between $C_{A}$ and $Q_{\max }$ for $1 \mathrm{D}$ and $2 \mathrm{D}$ results correspond to possible values of the localizable entanglement. Parameters for the 1D MPS calculation: $m=20$. Parameters for the 2D TERG calculation: $D=4, D_{c}=20$

tion $C_{F}$ and negativity $N$. Our TN results are in a very good agreement with the Monte-Carlo results by Syljuasen [11]. Of course, calculations close to the critical point in $2 \mathrm{D}$ are difficult both for $\mathrm{TN}$ and MC methods, but, clearly, the cusp at the critical point can be better resolved with the $\mathrm{TN}$ method used here. Close to the critical point, the MC results of Ref. [11] are very noisy. The concurrence of forma- tion for 1D does not peak at the critical point, but shows an inflection. This is in agreement with the analytical results presented in Ref. [7].

The negativity shows similar characteristics as the concurrence of formation both in 1D and 2D. Concurrence of formation and negativity reach their maximum at the same value for the magnetic field.

The local entanglement $S_{\text {loc }}$ shown in Fig. 3 is the simplest form of a block-block entanglement, the entanglement between two neighboring spins and their environment. We see that, both in $1 \mathrm{D}$ and 2D, this measure has a peak with a cusp at the critical point. Not surprisingly, the absolute value of $S_{\mathrm{loc}}$ at the critical point is the largest among other entanglement measures we calculate from the two-site reduced density matrix. This is due to the fact that $S_{\text {loc }}$ corresponds to the entanglement between two neighboring spins as one party with all other spins as another party in contrast to the entanglement between just two neighbor spins in the case of $C_{F}, N, C_{A}$. Similar to the one-site entanglement entropy, $S_{\text {loc }}$ is small in the ferromagnetic phase $\left(h<h_{\mathrm{cr}}\right)$, increases sharply close to the critical point, and then decreases slowly in the paramagnetic phase $\left(h>h_{\mathrm{cr}}\right)$.

Figure 3 also demonstrates that the bipartite entanglement per bond identifies the critical point having a peak with cusp there. This measure exemplifies one useful advantage of the translationally invariant TN methods in 2D: the possibility to extract information about the state right from the $\mathrm{TN}$ representation, i.e. one does not need to calculate expectation values at potentially high numerical cost.

In Fig. 4, we compare the upper bound (concurrence of assistance $C_{A}$ ) and lower bound (maximal two-site correlation function $Q_{\max }$ ) of the localizable entanglement as a function of the magnetic field. Our results show cusps at the critical point and are in a good agreement with those obtained within other methods $[11,55]$. Note that our MPS and PEPS implementations intrinsically break the $Z_{2}$ symmetry, thus leading to product states for small and large magnetic fields.

All entanglement measures discussed above are able to identify the critical point of the system both in $1 \mathrm{D}$ and $2 \mathrm{D}$. The fastest and easiest way to identify the critical point is obtained from the entanglement per bond. This measure explicitly requires a tensor network representation and cannot be obtained using other methods. As expected, all entanglement mea- 
sures approach zero for small and large transverse magnetic fields, which indicates product states for these limits.

In Fig. 5, we show the NN entanglement given in terms of the concurrence of assistance $2\left[C_{A}^{1 \mathrm{D}}\right]_{\mathrm{NN}}^{2}$, the one-tangle $\tau_{1}^{1 \mathrm{D}}$, and the NN entanglement given as a function of the concurrence of formation $2\left[C_{F}^{1 \mathrm{D}}\right]_{\mathrm{NN}}^{2}$ for the $1 \mathrm{D}$ quantum Ising model. By comparing $\tau_{1}^{1 \mathrm{D}}$ and $2\left[C_{F}^{1 \mathrm{D}}\right]_{\mathrm{NN}}^{2}$, we see that the CKW inequality (3) is fulfilled, and the nearest-neighbor two-particle entanglement corresponds to only about $25 \%$ of the total entanglement in the critical region given by $\tau_{1}$. At the same time, we relate the residual $75 \%$ of entanglement to long-range and many-way entanglement. This behavior is a quantitative evidence that the phase transition is characterized by the presence of a complicated entanglement structure in the state. Furthermore, outside of the critical region, $2\left[C_{F}^{1 \mathrm{D}}\right]_{\mathrm{NN}}^{2}$ nearly exhausts the $\mathrm{CKW}$ inequality. Comparing $\tau_{1}^{1 \mathrm{D}}$ and $2\left[C_{A}^{1 \mathrm{D}}\right]_{\mathrm{NN}}^{2}$, we conclude that already the nearest neighbor entanglement contributions are larger than the lower bound $\tau_{1}^{1 \mathrm{D}}$ of how much entanglement can be created by assistance.

Figure 6 displays the entanglement monogamy analysis for the 2D quantum Ising model. Here, we compare $4\left[C_{A}^{2 \mathrm{D}}\right]_{\mathrm{NN}}^{2}, \tau_{1}^{2 \mathrm{D}}$ and $4\left[C_{F}^{2 \mathrm{D}}\right]_{\mathrm{NN}}^{2}$. The CKW inequality is fulfilled, and the nearest-neighbor entanglement in the critical region corresponds to about $50 \%$ of the total entanglement. In comparison to the $1 \mathrm{D}$ result, we observe that the $2 \mathrm{D}$ nearest-neighbor entanglement has bigger weight in the entanglement distribution, which can be explained by the presence of a larger number of nearest neighbors of each site. Again, similar to the $1 \mathrm{D}$ case, $4\left[C_{F}^{1 \mathrm{D}}\right]_{\mathrm{NN}}^{2}$ nearly exhausts the $\mathrm{CKW}$ inequality outside of the critical region. Again, comparing $\tau_{1}^{2 \mathrm{D}}$ and $4\left[C_{A}^{1 \mathrm{D}}\right]_{\mathrm{NN}}^{2}$, we see that the nearest-neighbor entanglement terms in $2 \mathrm{D}$ in general are also already larger than the lower bound $\tau_{1}^{2 \mathrm{D}}$ on how much entanglement can be created by assistance.

\section{2. $X X Z$ model}

Next, we study the spin- $\frac{1}{2}$ XXZ (anisotropic Heisenberg) model,

$H^{\mathrm{XXZ}}=\sum_{\langle i, j\rangle}\left\{\sigma_{i}^{x} \otimes \sigma_{j}^{x}+\sigma_{i}^{y} \otimes \sigma_{j}^{y}+\Delta \sigma_{i}^{z} \otimes \sigma_{j}^{z}\right\}$

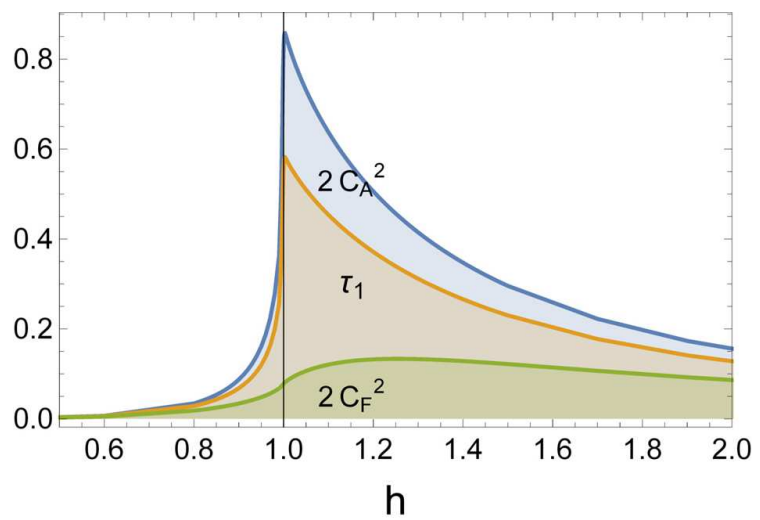

Fig. 5. Entanglement monogamy analysis for the 1D quantum Ising model: comparison of the concurrence of formation $C_{F}$, the concurrence of assistance $C_{A}$ and the 1-tangle $\tau_{1}$. For details, see the discussion in the main text. Parameters for the 1D MPS calculation: $m=20$

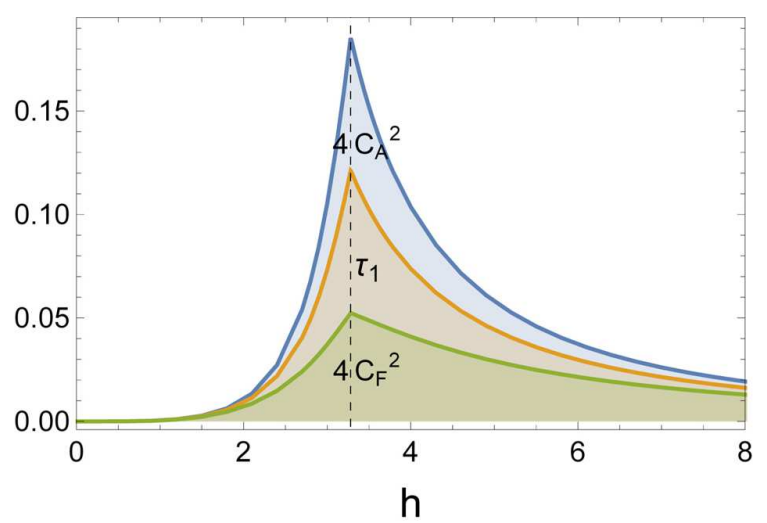

Fig. 6. Entanglement monogamy analysis for the $2 \mathrm{D}$ quantum Ising model: comparison of the concurrence of formation $C_{F}$, the concurrence of assistance $C_{A}$ and the 1-tangle $\tau_{1}$. For details, see the discussion in the main text. Parameters for the 2D TERG calculation: $D=4, D_{c}=20$. Critical value of the magnetic field is $h_{\mathrm{cr}} \approx 3.28$

as a function of the anisotropy parameter $\Delta$. The Hamiltonian of this model is U(1)-symmetric (corresponding to an invariance under a $\mathrm{U}(1)$ rotation about the spin $z$ axis), as well as $Z_{2}$-symmetric (corresponding to an invariance under a $\pi$ rotation about the spin $x$ or $y$ axis). It is $\mathrm{SU}(2)$-symmetric at the Heisenberg point $\Delta=1$. The ground state of the XXZ model in different phases preserves these symmetries not depending on the space dimension [16]. The $Z_{2}$ symmetry implies that $\left\langle\sigma_{i}^{z}\right\rangle=0$ and $\left\langle\sigma_{i}^{x} \sigma_{j}^{z}\right\rangle=\left\langle\sigma_{i}^{y} \sigma_{j}^{z}\right\rangle=0$. 


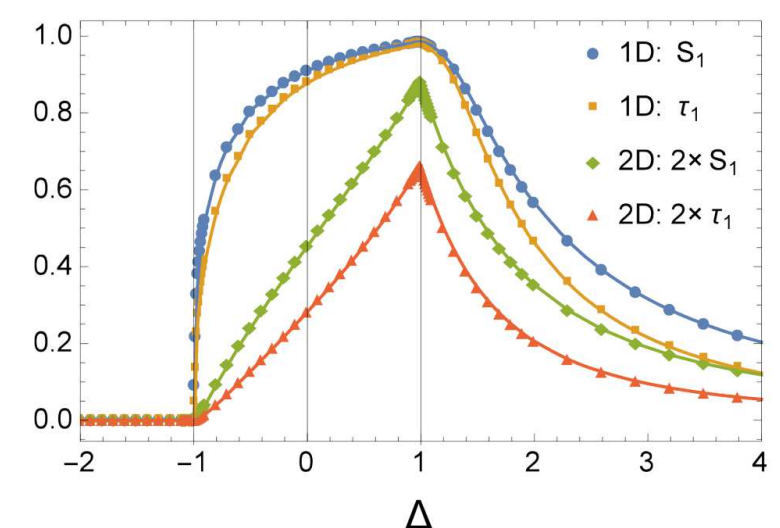

Fig. 7. Comparison of the one-site entanglement entropy $S_{1}$ and the one-tangle $\tau_{1}$ dependence on $\Delta$ for the 1D and 2D XXZ models. Results for 2D model are multiplied by a factor of 2 . Parameters for the 1D MPS calculation: $m=20$. Parameters for the 2D TERG calculation: $D=4, D_{c}=20$

The U(1) symmetry implies that $\left\langle\sigma_{i}^{x}\right\rangle=\left\langle\sigma_{i}^{y}\right\rangle=0$, $\left\langle\sigma_{i}^{x} \sigma_{j}^{x}\right\rangle=\left\langle\sigma_{i}^{y} \sigma_{j}^{y}\right\rangle,\left\langle\sigma_{i}^{x} \sigma_{j}^{y}\right\rangle=0$.

The XXZ model has a richer phase structure than the Ising model: the $1 \mathrm{D} \mathrm{XXZ} \mathrm{model} \mathrm{shows} \mathrm{three}$ phases [56]. For $\Delta>1$, the system is in a gapped antiferromagnetic phase (in particular, it corresponds to a classical Ising antiferromagnet for large positive $\Delta$ ). At $\Delta=1$, there is a critical point, where an infinite-order Kosterlitz-Thouless quantum phase transition occurs from the antiferromagnetic phase to the XY phase. The system is equivalent here to the spin- $\frac{1}{2}$ Heisenberg antiferromagnet with a gapless ground state. In the XY phase $(|\Delta|<1)$, the system is gapless, and the correlation functions decay polynomially. At $\Delta=-1$, the system undergoes a first-order quantum phase transition to a ferromagnetic gapped phase for $\Delta<-1$. For large negative $\Delta$, the system resembles an Ising ferromagnet.

In the thermodynamic limit, the spontaneous $Z_{2}$ symmetry breaking occurs in the ferromagnetic $(\Delta<-1)$ and antiferromagnetic phases $(\Delta>1)$, but $Z_{2}$ symmetry is preserved in the $\mathrm{XY}$ phase. The continuous $U(1)$ symmetry remains unbroken in all three phases of the 1D XXZ model at zero temperature, as is required by the Mermin-Wagner theorem [57].

The two-dimensional XXZ model shows three different phases, as well $[58,59]$ : an antiferromagnetic phase for $\Delta>1$, an XY phase for $|\Delta|<1$, and a ferromagnetic phase for $\Delta<-1$. It undergoes a second-order phase transition at $\Delta=1[60]$ and a first-order phase transition at $\Delta=-1$ [61]. Just as in $1 \mathrm{D}$, the $Z_{2}$ symmetry is spontaneously broken in the ferromagnetic $(\Delta<-1)$ and antiferromagnetic phases $(\Delta>1)$ and remains unbroken in the $\mathrm{XY}$ phase. However, unlike in the $1 \mathrm{D}$ case, the continuous $\mathrm{U}(1)$ symmetry can be broken in the XY phase of the 2D XXZ model at zero temperature.

Numerical results for the magnetization (not presented in the text) show the U(1) symmetry breaking in the XY phase not only for the 2D model, as expected, but also for the $1 \mathrm{D}$ model. We observed that this $U(1)$ symmetry breaking in $1 \mathrm{D}$ is dependent on the chosen $m$ and gets slightly smaller with increasing $m$. It appears that one needs to use a code, which implements the $\mathrm{U}(1)$ symmetry of the states from the outset in order to get more precise results. We will do this in a future project. This nonphysical breaking of the $U(1)$ symmetry will be seen as well later in various calculated entanglement measures. Note that the translationally invariant MPS algorithm nicely obtains the antiferromagnetic phase, despite the fact that it uses equal tensors at each site.

In Fig. 7, we show the one-site entanglement measures: one-site entanglement entropy $S_{1}$ and onetangle $\tau_{1}$ for the one- and two-dimensional XXZ models. All these measures peak in cusps at the critical point $\Delta=1$ and are zero for the $\Delta<-1$. At the Heisenberg point in the 1D model, the ground state is $\mathrm{SU}(2)$-symmetric, and the one-site measures $S_{1}$ and $\tau_{1}$ approach their maximal possible values. Theoretically, it is expected that these quantities are equal to 1 throughout the XY phase in $1 \mathrm{D}$, but, due to the $\mathrm{U}(1)$ symmetry breaking present in the algorithms (as was mentioned) above, these quantities decrease while approaching the $\Delta=-1$ critical point. Again in $1 \mathrm{D}$, we would obtain better results for larger $m$ or by using a code, which respects the $\mathrm{U}(1)$ symmetry from the outset.

In Fig. 8, we show the two-site entanglement measures: concurrence of formation $C_{F}$ and negativity $N$ for the one- and two-dimensional XXZ models. The concurrence of formation for the $1 \mathrm{D}$ and $2 \mathrm{D}$ XXZ models was studied in Refs. [11, 12, 16], and our results are in a very good agreement. The figure nicely shows that $C_{F}$ and $N$ in one and two dimensions have maxima exactly at the critical point $\Delta=1$. It is known that $C_{F}$ is related to the ground-state energy [62], and our results correspond to the fact discussed in Ref. [59] that the ground-state energy of 


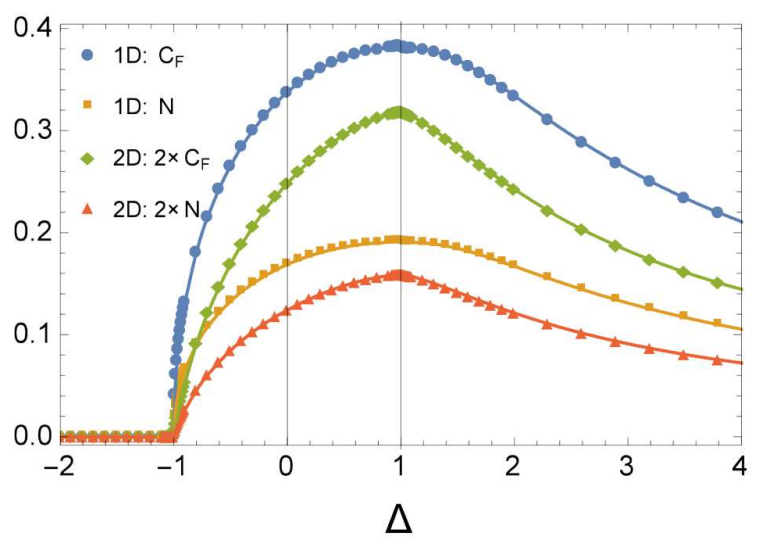

Fig. 8. Concurrence of formation $C_{F}$ and negativity $N$ as a function of $\Delta$ for the 1D and 2D XXZ models. Results for the 2D model are multiplied by a factor of 2 . Parameters for the 1D MPS calculation: $m=20$. Parameters for the 2D TERG calculation: $D=4, D_{c}=20$

the XXZ model in two and three dimensions shows a cusp at the transition point, thus leading to a cusp in the concurrence of formation. The $1 \mathrm{D} C_{F}$ and $N$ just have maxima at the critical point $\Delta=1$ without cusps.

Negativity for the XXZ model was previously studied for a two-qubit chain [63] and for infinite tree tensor network states [64]. Our results extend such studies to infinite chains and infinite square-lattice systems. Similarly to the quantum Ising model, we find that the negativities for the $1 \mathrm{D}$ and $2 \mathrm{D}$ XXZ models have a similar behavior as the concurrence of formation in $1 \mathrm{D}$ and $2 \mathrm{D}$. The $2 \mathrm{D}$ negativity peaks in a cusp, and the 1D negativity just shows maximum at the critical point $\Delta=1$.

In Fig. 9, we present the local entanglement $S_{\text {loc }}$ for the one- and two-dimensional XXZ models. The entanglement per bond $S_{\mathrm{PB}}$ for the $\mathrm{XXZ}$ model is also shown. Local entanglement for the 2D XXZ model was studied in [13]. However, $S_{\text {loc }}$ requires comment: for $\Delta \gg 1$, we observe that the local entanglement reported here approaches zero, while it approaches 1 in Ref. [13]. The reason for this difference is the fact that the ground state we consider here has broken $Z_{2}$ symmetry, while the authors of Ref. [13] assume that the ground state is $Z_{2}$-symmetric. We see that the local entanglements for $1 \mathrm{D}$ and $2 \mathrm{D}$ have similar behavior as the one-site entanglement entropy $S_{1}$. In both $1 \mathrm{D}$ and $2 \mathrm{D}, S_{\mathrm{loc}}$ vanishes at $\Delta=-1$ and peaks in a cusp at $\Delta=1$.

ISSN 2071-0194. Ukr. J. Phys. 2016. Vol. 61, No. 7

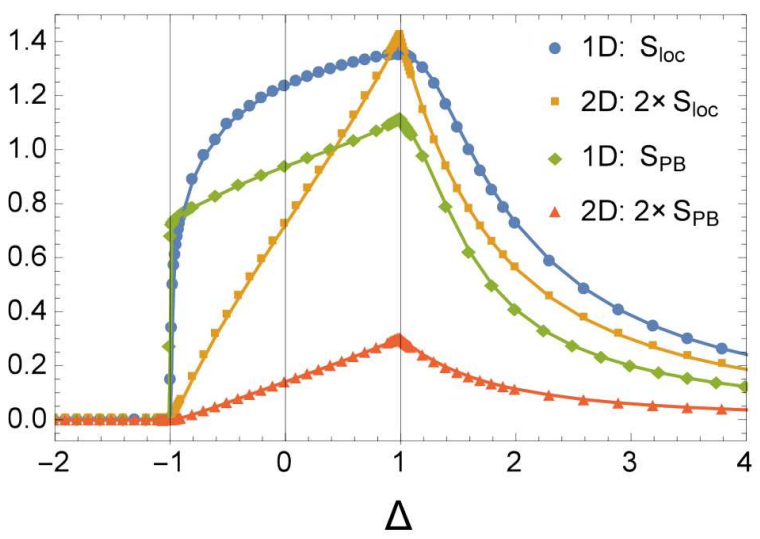

Fig. 9. Local entanglement $S_{\text {loc }}$ and entanglement per bond $S_{\mathrm{PB}}$ as a function of $\Delta$ for $1 \mathrm{D}$ and $2 \mathrm{D}$ XXZ models. Results for $2 \mathrm{D}$ model are multiplied by a factor of 2 . Parameters for MPS calculation: $m=20$. Parameters for TERG calculation: $D=4, D_{c}=20$

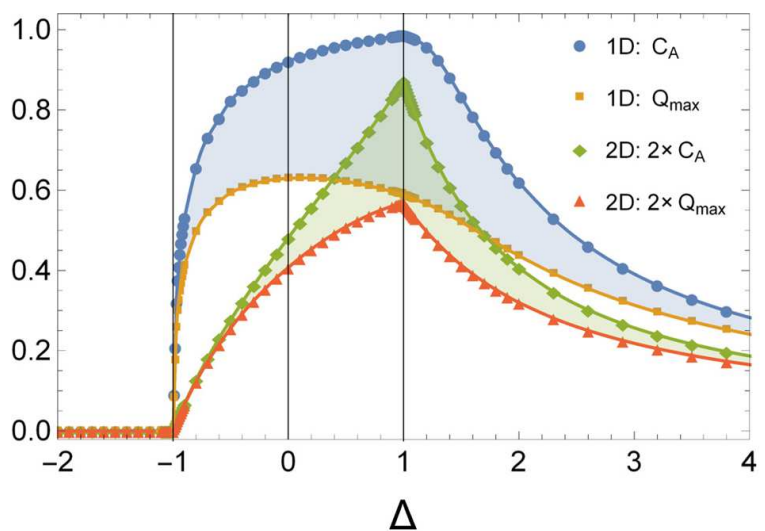

Fig. 10. Bounds on the localizable entanglement as a function of $\Delta$ for the 1D and 2D XXZ models. Results for the 2D model are multiplied by a factor of 2 . The shaded areas between $C_{A}$ and $Q_{\max }$ for $1 \mathrm{D}$ and $2 \mathrm{D}$ results correspond to the possible values of localizable entanglement. Parameters for the 1D MPS calculation: $m=20$. Parameters for the 2D TERG calculation: $D=4, D_{c}=20$

Entanglement per bond for the 2D XXZ model was analyzed in [14], but the authors discuss the $S_{\mathrm{PB}}$ dependence on an external magnetic field with some fixed $\Delta$. In our studies, we have no external magnetic field and vary the anisotropy parameter $\Delta$. Similarly to the quantum Ising model, $S_{\mathrm{PB}}$ shows its ability to determine critical points by vanishing at $\Delta=-1$ and having a peak with a cusp at $\Delta=1$.

In Fig. 10, we show the upper bound (concurrence of assistance $C_{A}$ ) and lower bound (maximal two-site correlation function $Q_{\max }$ ) on the localizable entan- 


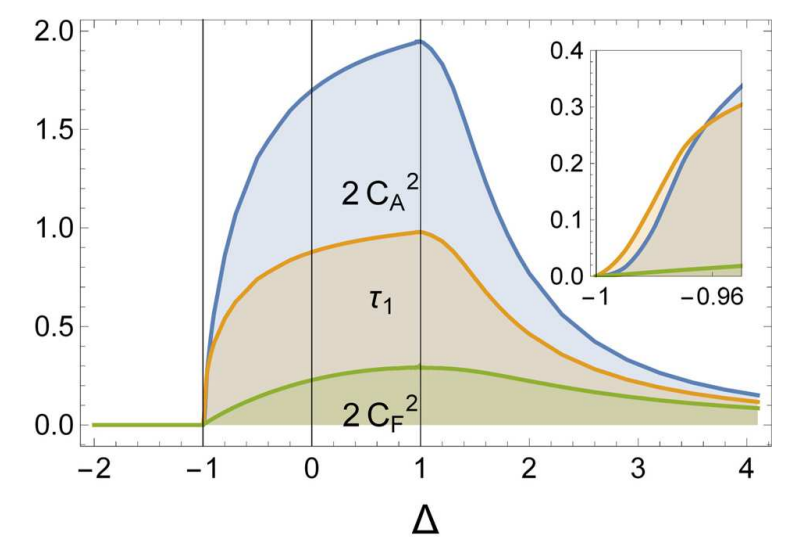

Fig. 11. Entanglement monogamy analysis for the $1 \mathrm{D} X X Z$ model: comparison of the concurrence of formation $C_{F}$, the concurrence of assistance $C_{A}$, and the 1-tangle $\tau_{1}$. For details, see the discussion in the main text. Parameters for the $1 \mathrm{D}$ MPS calculation: $m=20$

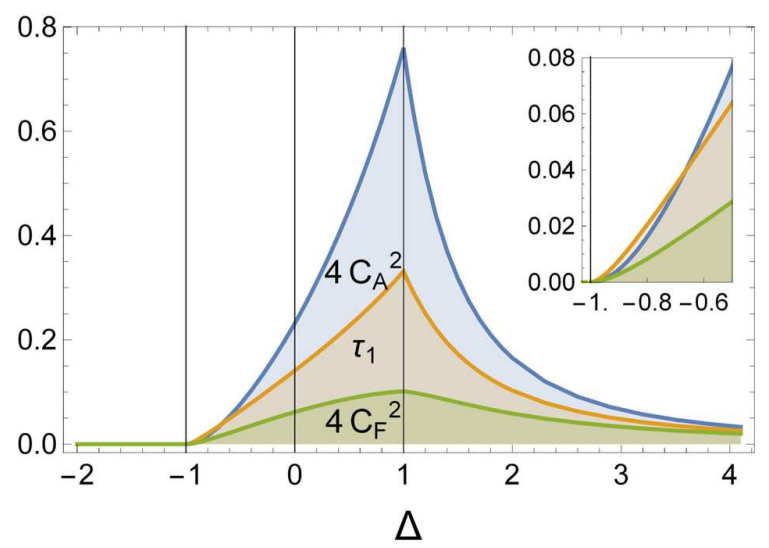

Fig. 12. Entanglement monogamy analysis for the $2 \mathrm{D} X X Z$ model: comparison of the concurrence of formation $C_{F}$, the concurrence of assistance $C_{A}$, and the 1-tangle $\tau_{1}$. For details, see the discussion in the main text. Parameters for the $2 \mathrm{D}$ TERG calculation: $D=4, D_{c}=20$

glement for the one- and two-dimensional XXZ models. These bounds in the two-dimensional case were also studied in Ref. [11].

We observe that, for $|\Delta|<1$, the concurrence of assistance $C_{A}$ and the two-point correlation function $Q_{\max }$ decrease for smaller $\Delta$, while $C_{A}=1$ throughout the XY phase, and $Q_{\max }$ does not drop to zero at one of the critical points in Ref. [11]. This difference between our results and those of Ref. [11] can be explained as follows: it was shown in Ref. [16] that the concurrence of formation $C_{F}$ is unaffected by the spontaneous $\mathrm{U}(1)$ symmetry breaking for the zero-field XXZ-model. Let us consider also the concurrence of assistance $C_{A}$. The formula for $C_{A}$ for maintained $\mathrm{U}(1)$ symmetry and broken $Z_{2}$ symmetry was introduced in $[11,17]$ :

$$
\begin{aligned}
& C_{A}=\frac{1}{2} \sqrt{\left(1+\left\langle\sigma_{i}^{z} \otimes \sigma_{j}^{z}\right\rangle\right)^{2}-\left\langle\sigma_{i}^{z}+\sigma_{j}^{z}\right\rangle^{2}}+ \\
& +\frac{1}{2} \sqrt{\left(1-\left\langle\sigma_{i}^{z} \otimes \sigma_{j}^{z}\right\rangle\right)^{2}-\left\langle\sigma_{i}^{z}-\sigma_{j}^{z}\right\rangle^{2}} .
\end{aligned}
$$

Following Ref. [16], we find $C_{A}$ for broken U(1) symmetry and maintained $Z_{2}$ symmetry:

$$
\begin{aligned}
& C_{A}= \\
& =\frac{1}{2}\left(\sqrt{\left(1+\left\langle\sigma_{i}^{x} \otimes \sigma_{j}^{x}\right\rangle\right)^{2}-4\left\langle\sigma_{i}^{x}\right\rangle^{2}}+1-\left\langle\sigma_{i}^{x} \otimes \sigma_{j}^{x}\right\rangle\right) .
\end{aligned}
$$

Obviously, $C_{A}$ (unlike $C_{F}$ ) is affected by the $\mathrm{U}(1)$ symmetry breaking, as it contains $\left\langle\sigma_{i}^{x}\right\rangle$.

When $\mathrm{U}(1)$ and $Z_{2}$ symmetries are obeyed, $C_{A}=$ $=1$. This should hold, e.g., for the Heisenberg point $\Delta=1$. We see from our $1 \mathrm{D}$ results that, indeed, $C_{A}(\Delta=1) \approx 1$. At the same time, our $2 \mathrm{D}$ results for $C_{A}$ for $\Delta=1$ do not reach the value $C_{A}=1$. This can be explained by the fact that it is numerically hard to converge to the point, where both $\left\langle\sigma_{i}^{x}\right\rangle$ and $\left\langle\sigma_{i}^{z}\right\rangle$ are zero, thus giving $C_{A}=1$ from both equations (11) and (12).

The discrepancy of our result for $Q_{\max }$ and the corresponding result from Ref. [11] can be explained by the $\mathrm{U}(1)$ symmetry breaking, resulting in a nonzero $\left\langle\sigma_{i}^{x}\right\rangle$. While $\left\langle\sigma_{i}^{x}\right\rangle$ increases, the function $Q^{x x}=$ $=\left\langle\sigma_{i}^{x} \sigma_{j}^{x}\right\rangle-\left\langle\sigma_{i}^{x}\right\rangle\left\langle\sigma_{j}^{x}\right\rangle$ (which is larger than $Q^{y y}$ and $Q^{z z}$ in the XY phase) decreases to zero.

Thus, we see that all entanglement measures discussed above are zero for $\Delta<-1$ and also approach zero for large positive $\Delta$, indicating a product state in this limit.

For the monogamy analysis in Fig. 11, we represent the nearest neighbor entanglement, given in terms of the concurrence of assistance $2\left[C_{A}^{1 \mathrm{D}}\right]_{\mathrm{NN}}^{2}$, one-tangle $\tau_{1}^{1 \mathrm{D}}$, and nearest neighbor entanglement given by the concurrence of formation $2\left[C_{F}^{1 \mathrm{D}}\right]_{\mathrm{NN}}^{2}$. For XXZ model, we are interested in two regions: the region to the right of $\Delta=-1$ and the region around $\Delta=1$. Comparing $\tau_{1}^{1 \mathrm{D}}$ and $2\left[C_{F}^{1 \mathrm{D}}\right]_{\mathrm{NN}}^{2}$, we see that the CKW inequality is fulfilled for all values of anisotropy parameter $\Delta$.

ISSN 2071-0194. Ukr. J. Phys. 2016. Vol. 61, No. 7 
The inset in Fig. 11 shows that the NN entanglement $2\left[C_{F}^{1 \mathrm{D}}\right]_{\mathrm{NN}}^{2}$ to the right of $\Delta=-1$ is very small in comparison to the $\tau_{1}$ value. We suggest that the long-range and many-way entanglement plays the major role in the entanglement distribution in this region. In the critical region around $\Delta=1$, the nearest neighbor two-particle entanglement $2\left[C_{F}^{1 \mathrm{D}}\right]_{\mathrm{NN}}^{2}$ contributes about $1 / 3$ of the total entanglement $\tau_{1}^{1 \mathrm{D}}$. For large $\Delta \gg 1$, the nearest neighbor two-particle entanglement approaches $\tau_{1}^{1 \mathrm{D}}$, by signaling that the many-way and long-range entanglement significantly decreases.

Thus, our results can be treated as a quantitative evidence that the phase transitions are characterized by the presence of a complicated, including long-range and many-way, entanglement structure of the states.

By comparing $\tau_{1}^{1 \mathrm{D}}$ and $2\left[C_{A}^{1 \mathrm{D}}\right]_{\mathrm{NN}}^{2}$, we see that the nearest neighbor entanglement in general is larger than the lower bound $\tau_{1}^{1 \mathrm{D}}$ on how much entanglement can be created by assistance. Only in the XY phase in the region close to $\Delta=-1$, the nearest neighbor entanglement does not exceed the $\tau_{1}^{1 \mathrm{D}}$. This feature is shown in the inset in Fig. 11.

Figure 12 shows the entanglement monogamy analysis for the 2D XXZ model. In this case, we compare $4\left[C_{A}^{2 \mathrm{D}}\right]_{\mathrm{NN}}^{2}, \tau_{1}^{2 \mathrm{D}}$ and $4\left[C_{F}^{2 \mathrm{D}}\right]_{\mathrm{NN}}^{2}$. Again, by comparing $\tau_{1}^{1 \mathrm{D}}$ and $2\left[C_{F}^{1 \mathrm{D}}\right]_{\mathrm{NN}}^{2}$, we see that the CKW inequality is fulfilled for all values of anisotropy parameter $\Delta$. This indicates the consistency of our numerical data.

The inset in Fig. 12 shows that the NN entanglement $2\left[C_{F}^{1 \mathrm{D}}\right]_{\mathrm{NN}}^{2}$ in the region to the right of $\Delta=-1$ contributes about $50 \%$ to $\tau_{1}$, by signaling that the short-range entanglement is rather large in comparison to the $1 \mathrm{D}$ model. In the critical region around $\Delta=1$, the nearest neighbor two-particle entanglement $2\left[C_{F}^{1 \mathrm{D}}\right]_{\mathrm{NN}}^{2}$ contributes about $1 / 3$ of the total entanglement $\tau_{1}^{1 \mathrm{D}}$. Similar to the $1 \mathrm{D}$ case, for large $\Delta \gg 1$, the nearest neighbor two-particle entanglement approaches $\tau_{1}^{2 \mathrm{D}}$.

Again, our results suggest that the phase transitions are characterized by the presence of a complicated entanglement structure of the states, including long-range and many-way.

By comparing $\tau_{1}^{2 \mathrm{D}}$ and $4\left[C_{A}^{1 \mathrm{D}}\right]_{\mathrm{NN}}^{2}$, we see that the nearest neighbor entanglement in general is larger than the lower bound $\tau_{1}^{2 \mathrm{D}}$ on how much entanglement can be created by assistance. Again, only in the $\mathrm{XY}$ phase in the region close to $\Delta=-1$, the nearest neighbor entanglement does not exceed $\tau_{1}^{1 \mathrm{D}}$, which is shown in the inset in Fig. 12.

\section{Conclusions}

We have investigated the entanglement properties of infinite $1 \mathrm{D}$ and $2 \mathrm{D}$ spin- $1 / 2$ systems, by using tensor network methods: the Ising model in transverse field and the anisotropic XXZ model.

Our analysis aimed at the understanding of quantum phases and QPTs in many-body systems, by using quantum information tools. This is based on the notion that every state (or phase) of a many-body system is characterized by a particular entanglement structure, which may have short-range, long-range, two-way (bipartite), three-way (tripartite), ..., multipartite constituents in different proportions. Some quantum phases may be characterized by an extremely complicated entanglement structure, while others just are product states (classical phases).

Different bipartite entanglement quantifiers such as the one-site entanglement entropy and the onetangle, the concurrence of formation and the negativity, the bounds on localizable entanglement (the concurrence of assistance and the two-point correlation function), the local entanglement, and the bipartite entanglement per bond are calculated and used in the analysis.

An interesting aspect we analyzed is the dependence of the entanglement structure on the particular symmetry in the ground state. Some measures appear to be invariant under symmetry breaking in the ground state and others not. We presented an analytical analysis for the concurrence of assistance, as similarly done in Ref. [16] for the concurrence of formation. We showed analytically that the concurrence of assistance depends on the U(1)-symmetry breaking and confirmed this numerically.

Our simulations for the 2D models confirm the observation [14] that the bipartite entanglement per bond can determine critical points successfully. This measure is unique for tensor network methods, because it is obtained not from the reduced density matrix, as other entanglement measures we use, but directly from the tensor network representation of the ground-state wave function. 
An intriguing analysis using a combination of two types of bipartite entanglement quantifiers, namely one-tangle and the concurrence of formation (or twotangle), was made. The numerical results obtained for both models and dimensions fulfill the Coffman-Kun$\mathrm{du}$-Wootters inequality for many spins. Through the entanglement monogamy property formulated within the Coffman-Kundu-Wootters inequality, we conclude that the obtained entanglement distribution indicateds the presence of a relatively large weight of either long-range or many-way entanglement in the critical region for both Ising and XXZ models in both dimensions. Our results are in a good agreement with ideas obtained via a similar analysis, but based on different analytical and numerical methods [9, 52].

We used TI MPS and TEBD for MPS in 1D, TERG and CTMRG with "simple update" for PEPS in $2 \mathrm{D}$ in order to model the ground states of the chosen models. Most of our results are in a good agreement with those obtained within theoretical predictions or other numerical methods, so we confirm the capability of the methods to model ground state wave functions. However, we observed that the TI MPS algorithm and the TEBD algorithm lead to a $\mathrm{U}(1)$ symmetry breaking in the $\mathrm{XY}$ phase for the 1D XXZ model, as soon as no symmetry conditions are imposed in our implementations from the outset. Therefore, our results are at variance with those assuming $\mathrm{U}(1)$ symmetry $[56,65]$.

Our work may be extended into several directions: for a more complete entanglement characterization of the models, it is important to consider other entanglement quantifiers and characteristics such as the fidelity [5], global entanglement [14], and tripartite entanglement [15]. An analysis of the quantum correlations beyond entanglement, e.g. the quantum discord [66], can be even more interesting.

A natural way to extend the monogamy analysis is to consider explicitly the long-ranged twoparticle entanglement terms and, moreover, to use the sharpened Coffman-Kundu-Wootters inequalities [47], e.g. by taking three-tangle terms into account [8]. Obtaining the three-tangle quantifier for a random mixed state is a nontrivial task, and special methods are needed for its calculation.

Mykhailo V. Rakov thanks Physikalisch-Technische Bundesanstalt for the financial support during short visits to Braunschweig.

\section{APPENDIX}

\section{Entanglement Measures}

In this appendix, we briefly review well-known definitions for various bipartite entanglement measures.

The first two are the one-site entanglement entropy $S_{1}$ and one-tangle $\tau_{1}$, which are obtained directly from the single-site reduced density matrix. The entanglement entropy [67] for bipartite pure states $\left|\psi_{12}\right\rangle$ is the von Neumann entropy of the reduced density matrix

$S\left(\left|\psi_{12}\right\rangle\right)=\mathcal{S}\left(\rho_{1}\right)=\mathcal{S}\left(\rho_{2}\right)$,

with the reduced density matrices $\rho_{1}=\operatorname{Tr}_{2}\left(\rho_{12}\right)$ and $\rho_{2}=$ $\operatorname{Tr}_{1}\left(\rho_{12}\right) ; \rho_{12}=\left|\psi_{12}\right\rangle\left\langle\psi_{12}\right|$, and $\operatorname{Tr}_{i}$ indicates a trace over the subsystem $i$. The von Neumann entropy $S$ of a density matrix $\rho$ is calculated from its eigenvalues $\lambda_{i}$ [67]:

$S(\rho)=-\rho \log _{2} \rho=-\sum_{i} \lambda_{i} \log _{2} \lambda_{i}$.

In the main text, we use $S_{1}=S\left(\rho_{1}\right)$. The one-tangle [8] is also calculated from the one-site reduced density matrix:

$\tau_{1}\left(\rho_{1}\right)=4 \operatorname{det} \rho_{1}$

Next, we mention measures obtained from the two-site reduced density matrix $\rho_{12}$. A simple measure of the bipartite entanglement in a mixed state is the entanglement of formation, $E_{F}$ [68]. It counts the minimum number of maximally entangled states (Bell states) needed to construct a given state, by using only local operations and classical communication (LOCC) (for details, see $[67,68]$ ). The entanglement of formation can be calculated from the concurrence of formation $C_{F}[69,70]:$

$E_{F}=h\left(\frac{1}{2}+\frac{\sqrt{1-C_{F}^{2}}}{2}\right)$,

where $h(x)$ denotes the binary entropy function. The concurrence of formation [70] is an entanglement measure for mixed states of two qubits defined as

$C_{F}(\rho)=\max \left(0, \lambda_{1}-\lambda_{2}-\lambda_{3}-\lambda_{4}\right)$,

where $\lambda_{1}, \lambda_{2}, \lambda_{3}, \lambda_{4}$ (in decreasing order) are the eigenvalues of the Hermitian matrix

$R=\sqrt{\sqrt{\rho_{12}} \tilde{\rho}_{12} \sqrt{\rho_{12}}}$

with $\tilde{\rho}_{12}=\left(\sigma_{y} \otimes \sigma_{y}\right) \rho_{12}^{*}\left(\sigma_{y} \otimes \sigma_{y}\right)$. Here, $\rho_{12}^{*}$ is the complex conjugate of the two-site density matrix $\rho_{12}$. Alternatively, $\lambda_{i}$ are the square roots of the eigenvalues of the non-Hermitian matrix $\rho_{12} \tilde{\rho}_{12}$. The concurrence is zero for a product state and one for a maximally entangled state.

Another type of concurrence, the concurrence of assistance $C_{A}$, was introduced in connection with the entanglement of assistance $E_{A}$ [71]. $C_{A}$ is obtained from [72]:

$C_{A}=\lambda_{1}+\lambda_{2}+\lambda_{3}+\lambda_{4}$.

ISSN 2071-0194. Ukr. J. Phys. 2016. Vol. 61, No. 7 
The entanglement of assistance measures the maximal bipartite entanglement which can be obtained, while doing measurements on the rest of spins. The idea of the entanglement of assistance originates from the analysis of tripartite systems described by a state $\left|\psi^{123}\right\rangle$. By varying the measurement on party 3, the "helper" 3 is able to influence the mixed state of parties 1 and 2 [73]. In order to use $E_{A}$ in practice, one must be able to perform a maximization over all different measurement strategies, thus this measure is difficult to be calculated. However, the following easily calculable bounds on $E_{A}$ exist: the upper bounds on $E_{A}$ are the entropic bound, fidelity bound, and concurrence bound $C_{A}$ [71]. The latter is used in the present publication.

The localizable entanglement $E_{L}[55]$ is defined as the maximal amount of entanglement that can be localized (on average) between two spins, while doing only local measurements on the rest of spins in the environment. $E_{L}$ cannot be obtained from the reduced density matrix alone, thus it is able to describe characteristics of the wave function that are not captured by two-point correlation functions, e.g. exotic phases like topological orders. The calculation of $E_{L}$ is not a trivial task, since one needs to optimize over all possible local measurement strategies. Nevertheless, it is possible to obtain bounds on $E_{L}$, by using only two-point correlation functions [55].

The upper bound for $E_{L}$ is the concurrence of assistance $C_{A}$, and the lower bound is obtained from the maximal two-point correlation function,

$\max \left(\left|Q_{12}^{x x}\right|,\left|Q_{12}^{y y}\right|,\left|Q_{12}^{z z}\right|\right) \leq E_{L} \leq C_{A}$,

where $Q_{12}^{\alpha \beta}(|\psi\rangle\langle\psi|)=\left\langle\psi\left|\sigma_{1}^{\alpha} \otimes \sigma_{2}^{\beta}\right| \psi\right\rangle-\left\langle\psi\left|\sigma_{1}^{\alpha} \otimes 1_{2}\right| \psi\right\rangle\langle\psi| 1_{1} \otimes$ $\sigma_{2}^{\beta}|\psi\rangle$, and $\sigma_{\alpha}$ are the Pauli spin matrices.

The negativity [74] is an "easy-to-compute" measure defined as

$\mathcal{N}\left(\rho_{12}\right)=\frac{\left\|\rho^{\Gamma_{1}}\right\|_{1}-1}{2}$,

where $\rho_{12}^{\Gamma_{1}}$ is the partially transposed density matrix $\rho_{12}$ with respect to subsystem $1,\left\|\rho_{12}\right\|_{1}=\operatorname{Tr} \sqrt{\rho_{12}^{\dagger} \rho_{12}}$ is the trace norm, and $\left\|\rho_{12}\right\|_{1}$ is calculated as a sum of the singular values of $\rho_{12}$.

A simple form of the bipartite entanglement is the entanglement between two neighboring spins and the other spins of the system. This measure is called local entanglement [13]. The two-site local entanglement $S_{\text {loc }}$ is obtained by tracing out all spin degrees of freedom of the system except the two nearestneighbour spins and then calculating the von Neumann entropy of the resulting reduced density matrix $\rho_{12}$,

$S_{\text {loc }}=S\left(\rho_{12}\right)$.

Another entanglement measure, which can be used if we have a tensor network representation of the state available in the conventional form, is the bipartite entanglement per bond $S_{\mathrm{PB}}$ [14]. It is obtained from the bond vectors [22] connecting two neighboring sites. The bond vectors contain essential entanglement information about the system. The entanglement per bond $S_{\mathrm{PB}}$ is given by

$S_{\mathrm{PB}}=-\sum_{i} \lambda_{i}^{2} \log _{2} \lambda_{i}^{2}$.

ISSN 2071-0194. Ukr. J. Phys. 2016. Vol. 61, No. 7 where the components of the bond vectors are normalized so that $\sum_{i} \lambda_{i}^{2}=1$.

Other entanglement measures like the fidelity [75], global entanglement [14], and entanglement spectrum [76] with Schmidt gap exist, which also can be used to analyze the entanglement in many-body systems. These measures are not considered in the present text.

1. S. Sachdev, Quantum Phase Transitions (Cambridge Univ. Press, Cambridge, 2011).

2. X.-G.Wen, Quantum Field Theory of Many-Body Systems (Oxford Univ. Press, New York, 2004).

3. H.T. Diep, Frustrated spin systems (World Scientific, Singapore, 2013).

4. T. Senthil, L. Balents, S. Sachdev, A. Vishwanath, and M.P.A.Fisher, Phys. Rev. B 70, 144407 (2004).

5. L. Amico, R. Fazio, A. Osterloh, and V. Vedral, Rev. Mod. Phys. 80, 517 (2008).

6. T. J. Osborne and M. A. Nielsen, Phys. Rev. A 66, 032110 (2002).

7. A. Osterloh, L. Amico, G. Falci, and R. Fazio, Nature 416, 608 (2002).

8. V. Coffman, J. Kundu, and W.K. Wootters, Phys. Rev. A 61, 052306 (2000).

9. L. Amico, F. Baroni, A. Fubini, D. Patanè, V. Tognetti, and P. Verrucchi, Phys. Rev. A 74, 022322 (2006).

10. M.B. Plenio and S. Virmani, Quant. Inf. Comput. 7, 1 (2007).

11. O.F. Syljuasen, Phys. Lett. A 322, 25 (2004).

12. S.-J. Gu, G.-S. Tian, and H.-Q. Lin, Phys. Rev. A 71, $052322(2005)$.

13. S.-J. Gu, G.-S. Tian, and H.-Q. Lin, New J. Phys. 8, 61 (2006).

14. C.-Y. Huang and F.-L. Lin, Phys. Rev. A 81, 032304 (2010).

15. J. Stasińska, B. Rogers, M. Paternostro, G. De Chiara, and A. Sanpera, Phys. Rev. A 89, 032330 (2014).

16. O.F. Syljuåsen, Phys. Rev. A 68, 060301 (2003).

17. A. Osterloh, G. Palacios, and S. Montangero, Phys. Rev. Lett. 97, 257201 (2006).

18. J.I. Cirac and F. Verstraete, J. Phys. A: Math. Theor. 42, 504004 (2009).

19. F. Verstraete, V. Murg, and J. Cirac, Adv. Phys. 57, 143 (2008).

20. R. Orús, Ann. Phys. 349, 117 (2014).

21. S.R. White, Phys. Rev. Lett. 69, 2863 (1992).

22. G. Vidal, Phys. Rev. Lett. 91, 147902 (2003).

23. F. Verstraete and J. Cirac (2004), cond-mat/0407066.

24. A. García-Sáez and J.I. Latorre, Phys. Rev. B 87, 085130 (2013).

25. H.C. Jiang, Z.Y. Weng, and T. Xiang, Phys. Rev. Lett. 101, 090603 (2008).

26. H.H. Zhao, Z.Y. Xie, Q.N. Chen, Z.C. Wei, J.W. Cai, and T. Xiang, Phys. Rev. B 81, 174411 (2010).

27. U. Schollwöck, Ann. Phys. 326, 96 (2011). 
28. Z.-C. Gu, M. Levin, and X.-G. Wen, Phys. Rev. B 78, 205116 (2008).

29. J. Jordan, R. Orús, G. Vidal, F. Verstraete, and J.I. Cirac, Phys. Rev. Lett. 101, 250602 (2008).

30. R. Orús and G. Vidal, Phys. Rev. B 80, 094403 (2009).

31. S. Östlund and S. Rommer, Phys. Rev. Lett. 75, 3537 (1995).

32. S. Rommer and S. Östlund, Phys. Rev. B 55, 2164 (1997).

33. G. Vidal, Phys. Rev. Lett. 98, 070201 (2007).

34. M. Levin and C.P. Nave, Phys. Rev. Lett. 99, 120601 (2007).

35. H.F. Trotter, Proc. Amer. Math. Soc. 10, 545 (1959).

36. W. Magnus, Commun. Pure App. Math. 7, 649 (1954).

37. G. Vidal, Phys. Rev. Lett. 93, 040502 (2004).

38. B. Pirvu, V. Murg, J.I. Cirac, and F. Verstraete, New J. Phys. 12, 025012 (2010).

39. W. Li, J. von Delft, and T. Xiang, Phys. Rev. B 86, 195137 (2012).

40. R.J. Baxter, J. Math. Phys. 9, 650 (1968).

41. T. Nishino and K. Okunishi, J. Phys. Soc. Jpn. 65, 891 (1996).

42. T. Nishino and K. Okunishi, J. Phys. Soc. Jpn. 66, 3040 (1997).

43. R. Orús, Phys. Rev. B 85, 205117 (2012).

44. X.-N. Zhu and S.-M. Fei, Phys. Rev. A 90, 024304 (2014).

45. G. Gour, S. Bandyopadhyay, and B.C. Sanders, J. Math. Phys. 48, 012108 (2007).

46. P. Rungta, V. Bužek, C.M. Caves, M. Hillery, and G.J. Milburn, Phys. Rev. A 64, 042315 (2001).

47. B. Regula, S. Di Martino, S. Lee, and G. Adesso, Phys. Rev. Lett. 113, 110501 (2014).

48. R. Horodecki, P. Horodecki, M. Horodecki, and K. Horodecki, Rev. Mod. Phys. 81, 865 (2009).

49. L. Amico, A. Osterloh, F. Plastina, R. Fazio, and G. Massimo Palma, Phys. Rev. A 69, 022304 (2004).

50. F. Baroni, A. Fubini, V. Tognetti, and P. Verrucchi, J. Phys. A: Math. Theor. 40, 9845 (2007).

51. T. Roscilde, P. Verrucchi, A. Fubini, S. Haas, and V. Tognetti, Phys. Rev. Lett. 94, 147208 (2005).

52. B. Li, S.-H. Li, and H.-Q. Zhou, Phys. Rev. E 79, 060101 (2009).

53. E. Lieb, T. Schultz, and D. Mattis, Ann. Phys. 16, 407 (1961).

54. H.W. J. Blöte and Y. Deng, Phys. Rev. E 66, 066110 (2002)

55. F. Verstraete, M. Popp, and J. I. Cirac, Phys. Rev. Lett. 92, 027901 (2004).

56. H.-J. Mikeska and A.K. Kolezhuk, in Quantum Magnetism, edited by U. Schollwöck, J. Richter, D.J. Farnell, and R.F. Bishop (Springer, Berlin, Heidelberg, 2004), p. 1.

57. N.D. Mermin and H. Wagner, Phys. Rev. Lett. 17, 1133 (1966).

58. S. Yunoki, Phys. Rev. B 65, 092402 (2002).

59. H.-Q. Lin, J.S. Flynn, and D.D. Betts, Phys. Rev. B 64 214411 (2001)

60. W.-L. You and Y.-L. Dong, Phys. Rev. B 84, 174426 (2011)
61. R.F. Bishop, D.J.J. Farnell, and J.B. Parkinson, J. Phys.: Cond. Matter 8, 11153 (1996).

62. L.-A.Wu, M.S. Sarandy, and D.A. Lidar, Phys. Rev. Lett. 93, 250404 (2004).

63. Q. Meng and T. Dong-Ping, Chinese Phys. C 33, 249-251 (2009).

64. G.-H. Liu, W. Li, W.-L. You, G. Su, and G.-S. Tian, EPL 101, 57001 (2013).

65. L. Justino and T. R. de Oliveira, Phys. Rev. A 85, 052128 (2012).

66. H. Ollivier and W. H. Zurek, Phys. Rev. Lett. 88, 017901 (2001).

67. M.A. Nielsen and I.L. Chuang, Quantum Computation and Quantum Information (Cambridge Univ. Press, Cambridge, 2000).

68. C.H. Bennett, D.P. DiVincenzo, J.A. Smolin, and W.K. Wootters, Phys. Rev. A 54, 3824 (1996).

69. S. Hill and W.K. Wootters, Phys. Rev. Lett. 78, 5022 (1997)

70. W.K. Wootters, Phys. Rev. Lett. 80, 2245 (1998).

71. D.P. DiVincenzo, C.A. Fuchs, H. Mabuchi, J.A. Smolin, A. Thapliyal, and A. Uhlmann, LNCS 1509, 247 (1999).

72. T. Laustsen, F. Verstraete, and S.J. van Enk, Quant. Inf. Comp. 3, 64 (2003).

73. J.A. Smolin, F. Verstraete, and A. Winter, Phys. Rev. A 72, 052317 (2005).

74. G. Vidal and R.F. Werner, Phys. Rev. A 65, 032314 (2002).

75. D. Schwandt, F. Alet, and S. Capponi, Phys. Rev. Lett. 103, 170501 (2009).

76. H. Li and F.D.M. Haldane, Phys. Rev. Lett. 101, 010504 (2008)

Received 14.02.16

Б. Брайорр-Орpс, М. Вайраух, М.В. Раков

ЧИСЕЛЬНІ ДОСЛІДЖЕННЯ

ВЛАСТИВОСТЕЙ ЗАПЛУТАНОСТІ

В КВАНТОВИХ МОДЕЛЯХ ІЗІНГА ТА ХХZ

Р е $з$ ю м е

Досліджуються властивості заплутаності в нескінченних одно- і двовимірних квантових моделях Ізінга та XXZ зі спіном $1 / 2$. Для моделювання основних станів моделей, що розглядаються, використовуються методи з тензорними мережами (матричнодобуткові стани (МДС) з трансляційною інваріантністю та проріджування блоків з еволюцією часу (ПБЕЧ) для МДС, ренормгрупа тензорної заплутаності (РГТЗ) та ренормгрупа кутових трансферних матриць (РГKTM) з "простим корегуванням" для проеційованих попарно заплутаних станів (ППЗС)). Різні міри заплутаності, якот: одночастинкова ентропія заплутаності, one-tangle, узгодження формування та підтримки, негативність та заплутаність на один зв'язок, порівнюються на предмет їхньої здатності відображати "структуру" фазових діаграм моделей (наприклад, фазові переходи). Вивчається зв'язок між симетрією основних станів та їхньою заплутаністю, а також через моногамність заплутаності аналізується заплутаність короткої та далекої дії. 